\title{
Desigualdades sanitarias y sociales en la salud materna y del niño en Paraguay
}

\author{
Edgar Tullo, ${ }^{1}$ María Jose Lerea, ${ }^{1}$ Rosa González, ${ }^{1}$ Julio Galeano, ${ }^{1}$ María Delasnieve Insfrán, ${ }^{2}$ \\ Mara Muñoz, ${ }^{3}$ Miguel Aragón ${ }^{3}$ y Antonio Sanhueza ${ }^{4}$
}

Forma de citar

Tullo E, Lerea MJ, González R, Galeano J, Insfrán MD, Muñoz M, et al. Desigualdades sanitarias y sociales en la salud materna y del niño en Paraguay. Rev Panam Salud Publica. 2020;44:e107. https://doi.org/10.26633/RPSP.2020.107

RESUMEN

Objetivo. Describir las desigualdades sanitarias y sociales en indicadores de salud materna y del niño definidos en las metas del Objetivo de Desarrollo Sostenible (ODS) 3.1 y ODS 3.2 a partir de datos administrativos, entre los departamentos de Paraguay en 2017.

Métodos. Diseño ecológico de carácter descriptivo cuantitativo. Se utilizaron medidas simples de brechas y medidas complejas de gradiente basadas en el ajuste de modelos de regresión binomial negativo y logístico. Resultados. Cincuenta por ciento de los departamentos de Paraguay tienen valores estimados de razón de mortalidad materna (RMM) mayores que el valor nacional. El porcentaje de partos atendidos por profesional calificado en el país alcanza 98,1\% con valores que fluctúan entre 82,4\% y 99,9\%. Hay 13 de 18 departamentos con valores de la tasa de mortalidad en menores de 5 años (TMM5) mayores que el promedio nacional, con un rango entre 4,2 y 49,2 muertes por cada 1000 nacidos vivos. Los valores de la tasa de mortalidad neonatal (TMN) en los departamentos varían entre 2,6 y 45,1 muertes por cada 1000 nacidos vivos. Existen grandes desigualdades sanitarias y sociales en la RMM, la TMM5 y la TMN entre los departamentos. No se detectan desigualdades elevadas en el porcentaje de partos atendidos por profesional calificado entre los departamentos.

Conclusiones. Paraguay debe hacer esfuerzos importantes para disminuir las desigualdades sanitarias y sociales que existen en la RMM, la TMM5 y la TNN entre los departamentos. Se deben establecer metas numéricas de mejoría de los valores nacionales y reducción de las desigualdades en estos indicadores, lo cual permitirá rendir cuentas sobre el compromiso de "no dejar a nadie atrás" establecido en los ODS, y ayudará a generar estrategias que permitan mejorar la salud de la mujer y el niño en Paraguay.

Palabras clave Desarrollo Sostenible; desigualdades en la salud; mortalidad materna; mortalidad neonatal; Paraguay.

El término desigualdad en salud se refiere a las diferencias observables en salud entre dos o más grupos socialmente determinados; el término inequidad hace referencia a un tipo específico de desigualdad que denota una diferencia injusta en la salud en el sentido que es arbitraria, innecesaria y evitable (1).

Los países de la Región de las Américas están prestando mayor atención a la medición de las desigualdades sociales en salud; por ejemplo, algunos países han realizado estudios de desigualdades sociales en indicadores de salud entre los niveles subnacionales (2). Medir las desigualdades sociales en salud permite evidenciar las diferencias injustas que existen en salud, y son, por lo tanto, la primera acción necesaria para diseñar estrategias que permitan reducir las brechas existentes entre los grupos más favorecidos y los menos favorecidos.

La Agenda 2030 para el Desarrollo Sostenible plantea el compromiso de garantizar que nadie se quede atrás, es decir,

\footnotetext{
Dirección General de Información Estratégica en Salud, Ministerio de Salud Pública y Bienestar Social, Paraguay. $\triangle$ Edgar Tullo, edgar.tullo@mspbs.gov.py 2 Dirección General de Problemas de Salud, Ministerio de Salud Pública y Bienestar Social, Paraguay.
} 
fortalece el concepto de equidad. Paraguay, al igual que otros países, ha asumido compromisos con la Agenda 2030 y los Objetivos de Desarrollo Sostenible (ODS). El ODS 3: Garantizar una vida sana y promover el bienestar de todos a todas las edades, tiene definidos 27 indicadores de salud, entre los cuales están los relacionados con las metas de salud materna y del niño (ODS 3.1 y ODS 3.2).

El artículo 48 de la Constitución Nacional de Paraguay establece el principio de igualdad y no discriminación, así como la responsabilidad del Estado para promover las condiciones y crear mecanismos adecuados para que la igualdad sea real y efectiva, allanando los obstáculos que impidan su ejercicio y facilitando la participación de la mujer en todos los ámbitos de la vida nacional (3). El acceso a servicios básicos como el saneamiento mejorado, agua segura y hogares con acceso a electricidad, avanzó de manera notable entre 2009 y 2018 en las zonas urbanas y rurales de Paraguay (4). La población en pobreza total a nivel nacional ha disminuido $33,5 \%$ en estos años (4). Con respecto al acceso a la salud en Paraguay en 2018, $26,9 \%$ de la población cuenta con seguro de salud y $71,0 \%$ de la población tiene acceso a los servicios de salud. Existe una diferencia marcada entre la población urbana, en la que 34,7\% cuenta con seguro de salud, en contraste con la población rural, cuyo porcentaje es de solo 14,3\% (4). Entre los indicadores de salud en Paraguay, la razón de muerte materna disminuyó $49,0 \%$ entre 2009 y 2014 (5), y al 2016 se reporta una razón de muerte materna de 86,4 por 100000 NV (6). Según datos recogidos desde el Subsistema de Información de Estadísticas Vitales, la tasa de mortalidad infantil también ha presentado un descenso importante de 65,4\% entre 1998 y 2017.

Paraguay cuenta con los resultados de la Encuesta de Indicadores Múltiples por Conglomerados (MICS, por sus siglas en inglés) de 2016 y años previos. Este tipo de encuestas es muy comparable en términos de muestreo y cuestionarios $(7,8)$. La Organización Mundial de la Salud (OMS) utiliza los resultados de estas encuestas para elaborar estudios de desigualdades en indicadores de salud por subpoblaciones, tales como por quintiles de riqueza y zona de residencia (urbana o rural). La OMS desarrolló la Herramienta de Evaluación de la Equidad Sanitaria (HEAT, por sus siglas en inglés) (9), que permite explorar desigualdades sociales en indicadores de salud por subpoblaciones en países. Sin embargo, los países cuentan con datos administrativos que se pueden utilizar para medir y monitorear las desigualdades sanitarias y sociales en indicadores de salud, como es el caso de los datos producidos por el Ministerio de Salud de Paraguay.

El objetivo de este trabajo es describir las desigualdades sanitarias y sociales en indicadores de salud materna y del niño definidos en las metas del ODS 3.1 y ODS 3.2 a partir de datos administrativos, entre los departamentos de Paraguay, en 2017.

\section{MATERIALES Y MÉTODOS}

El presente estudio tiene un diseño ecológico de carácter descriptivo cuantitativo. Se consideraron las siguientes variables:

- indicadores de salud: ODS 3.1.1 razón de mortalidad materna (RMM), ODS 3.1.2 porcentaje de partos atendidos por profesional calificado (PPAPC) que incluye médico, obstetra, enfermera y auxiliar; ODS 3.2.1 tasa de mortalidad en menores de 5 años (TMM5); y ODS 3.2.2 tasa de mortalidad neonatal (TMN), e
- indicadores sociales-socioeconómicos (estratificadores de equidad): porcentaje de acceso a saneamiento mejorado, porcentaje de pobreza total, promedio de años de estudio y tasa de desempleo abierto.

Se obtuvieron datos de los indicadores de salud y los estratificadores de equidad para cada uno de los 18 departamentos (la capital de Paraguay considerada como un departamento), desde los registros administrativos provenientes del Ministerio de Salud y la Encuesta Permanente de Hogares 2017 (10), respectivamente. Los indicadores de salud se calcularon con base en los datos de 2017 excepto la RMM, que se calculó en el quinquenio 2013-2018 con el objeto de estabilizar este indicador debido a la inestabilidad (cambios abruptos) de los valores en los departamentos.

En primer lugar, se llevó a cabo un análisis exploratorio de los datos para describir los estratificadores de equidad y los indicadores de salud a nivel nacional y en los departamentos. Esto incluyó un estudio de correlación entre los indicadores de salud y los estratificadores de equidad (no presentado aquí); este análisis condujo a los cuatro estratificadores de equidad más correlacionados con los indicadores de salud y que forman parte de esta investigación. Luego, se llevó a cabo un estudio de desigualdades en los indicadores de salud de los ODS 3.1 y ODS 3.2 para cada uno de los estratificadores de equidad (desigualdades sociales) como también para los mismos indicadores de salud (desigualdades sanitarias). En términos de desigualdades sociales simples, la brecha absoluta (BA) y la brecha relativa (BR) se obtienen al restar y dividir, respectivamente, el indicador de salud en el tercil más desfavorecido y el tercil más favorecido socialmente (11-13). Estos terciles se crearon al ordenar por el estratificador de equidad todo el panel de datos producido por los valores de los indicadores de salud, estratificador de equidad e indicador demográfico (en general, el denominador del indicador de salud) medidos en cada departamento. Si en vez de ordenar por un estratificador de equidad el panel de datos se hace por el indicador de salud, entonces se obtienen medidas simples de desigualdades sanitarias. Por otro lado, se consideraron medidas complejas en este trabajo, las cuales incluyen el índice de desigualdad absoluta binomial negativo (IDABN) y el índice de desigualdad relativa binomial negativo (IDRBN) para los indicadores de salud ODS 3.1.1, ODS 3.2.1 y ODS 3.2.2 que corresponden a razón y tasas de mortalidad. Para el indicador de salud ODS 3.1.2, se utiliza el índice de desigualdad absoluta logístico (IDAL) y el índice de desigualdad relativa logístico (IDRL). El IDABN y IDRBN se calculan al ajustar un modelo de regresión binomial negativo, metodología similar al utilizar un modelo de regresión de Poisson $(2,14)$. Se considera como variable de respuesta el número de muertes en cada departamento con la población al riesgo, y como variable independiente el valor del Ridit, que representa la posición social que tiene cada departamento y cuyos valores fluctúan entre 0 y 1 (2). Por otro lado, el IDAL y IDRL se obtienen al ajustar un modelo de regresión logístico de la siguiente forma:

$$
\ln (\mathrm{P} /(1-\mathrm{P}))=\mathrm{a}+\mathrm{b} \text { Ridit }
$$

donde $\mathrm{P}$ es la proporción de partos atendidos por profesional calificado en cada departamento, a (intercepto) y b (pendiente) son los parámetros del modelo y Ridit es la posición social que tiene cada departamento. El IDAL y el IDRL permiten comparar el indicador de salud entre los departamentos extremos, 
es decir para el valor del Ridit = RMin (departamento más desfavorecido socialmente) y el valor del Ridit = RMax (departamento más favorecido socialmente). Desde el modelo, esto es equivalente a comparar

$$
\ln (\mathrm{P} /(1-\mathrm{P}))=\mathrm{a}+\mathrm{b} \text { * } \mathrm{RMin}(\text { Ridit=RMin })
$$

con

$$
\ln (\mathrm{P} /(1-\mathrm{P}))=\mathrm{a}+\mathrm{b} \text { * RMax (Ridit=RMax })
$$

Al calcular la diferencia entre estos dos valores se produce el IDAL y al realizar el cociente entre estos valores se obtiene el IDRL.

\section{RESULTADOS}

El porcentaje de acceso a saneamiento mejorado en Paraguay alcanza un valor nacional de $82,7 \%$ en 2017 , el cual oscila entre $26,6 \%$ y $96,4 \%$ en los departamentos de Alto Paraguay y Central, respectivamente. El porcentaje de pobreza total nacional es de $25,8 \%$ en 2017 , con 12 departamentos sobre este valor y un rango de $11,6 \%$ en Asunción y de $47 \%$ en Caazapá. A nivel nacional, en 2017 hay un promedio de 8,9 años de estudio con solo dos departamentos sobre este promedio, donde el valor más bajo corresponde a Alto Paraguay (6,2 años) y el valor más alto a Asunción (11,0 años). La tasa de desempleo abierto en Paraguay es de 5,4, donde los tres departamentos con valores más altos en sus tasas son Alto Paraguay $(11,2)$, Presidente Hayes $(9,7)$ y Boquerón $(9,1)$.

La RMM estimada en Paraguay fue de 75,5 muertes maternas por 100000 NV en el quinquenio 2013-2017. Cincuenta por ciento de los departamentos tienen valores estimados de RMM mayores que el valor nacional; el departamento Caaguazú tiene la RMM más baja $(36,3)$ y el departamento Alto Paraguay tiene el valor más alto $(268,6)$ (figura 1). El PPAPC en el país es de $98,1 \%$ en 2017 , donde los departamentos con porcentajes más bajo y alto son Presidente Hayes (82,4\%) y Asunción (99,9\%), respectivamente. La TMM5 nacional fue de 14,5 muertes en menores de cinco años por $1000 \mathrm{NV}$ en 2017, con un rango entre 4,2 y 49,2 (una diferencia de 45 muertes); además, trece departamentos tienen valores mayores que el valor nacional. La TMN en Paraguay fue de 9,0 muertes neonatales por $1000 \mathrm{NV}$ en 2017 y varía entre 2,6 y 45,1 en los departamentos de Asunción y Alto Paraguay, respectivamente.

En el cuadro 1 se presentan los departamentos con mayor vulnerabilidad social y sanitaria; es decir, los departamentos con valores más altos (o más bajos) para cada uno de los

FIGURA 1. Distribución de la razón de mortalidad materna por cada 100000 nacidos vivos en los departamentos de Paraguay (2013-2017)

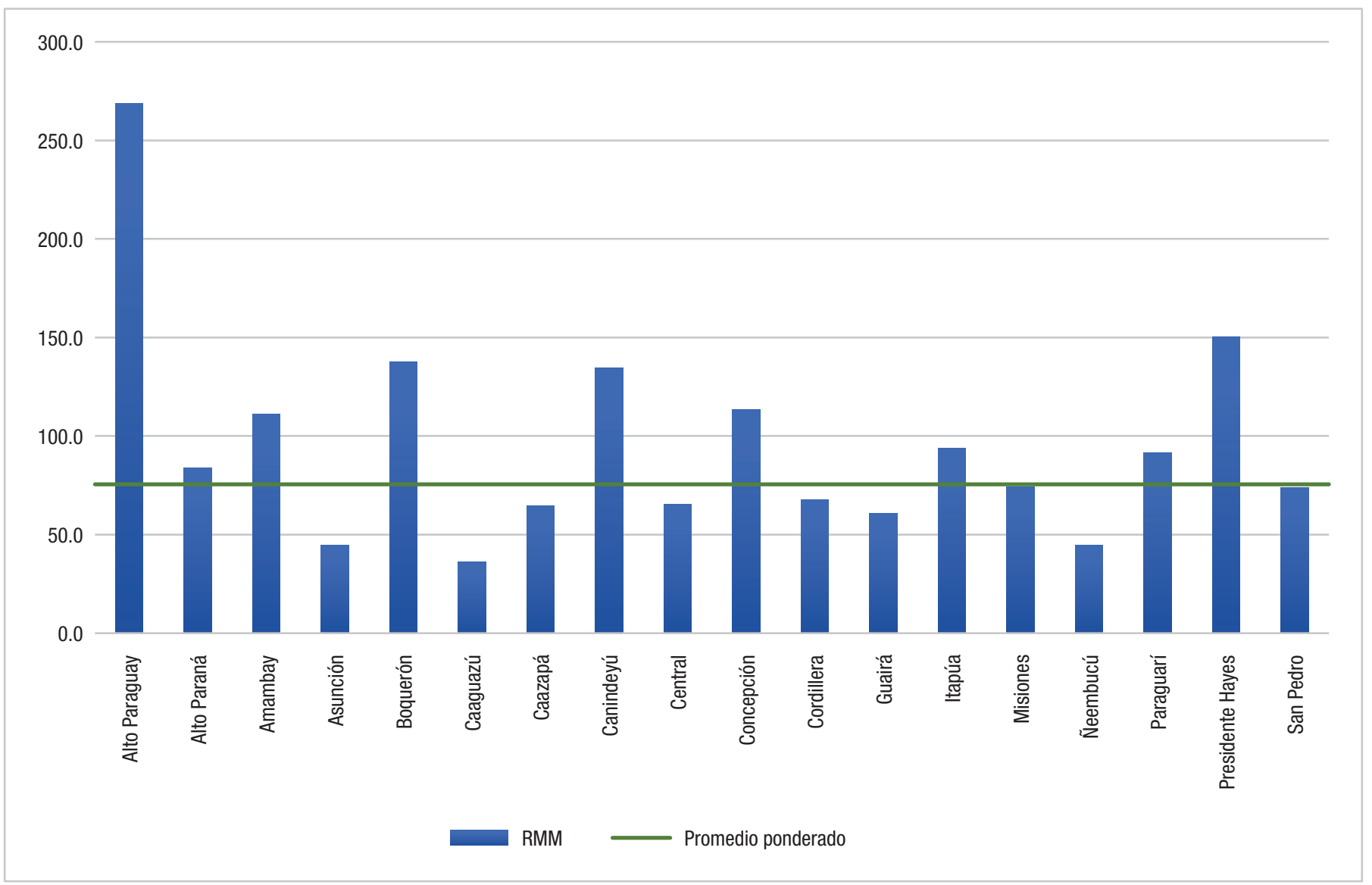


CUADRO 1. Departamentos de Paraguay con mayor grado de vulnerabilidad social y sanitaria, ordenados en grado decreciente de vulnerabilidad, 2017

\begin{tabular}{|c|c|c|c|c|c|c|c|c|c|c|c|c|c|c|c|}
\hline \multicolumn{2}{|c|}{$\begin{array}{l}\text { Acceso a } \\
\text { saneamiento } \\
\text { mejorado }\end{array}$} & \multicolumn{2}{|c|}{ Pobreza total } & \multicolumn{2}{|c|}{$\begin{array}{l}\text { Años de estudio } \\
\text { (promedio) }\end{array}$} & \multicolumn{2}{|c|}{$\begin{array}{l}\text { Tasa de } \\
\text { desempleo }\end{array}$} & \multicolumn{2}{|c|}{$\begin{array}{l}\text { Razón de mortalidad } \\
\text { materna }\end{array}$} & \multicolumn{2}{|c|}{$\begin{array}{l}\text { Tasa de mortalidad en } \\
\text { menores de } 5 \text { años }\end{array}$} & \multicolumn{2}{|c|}{$\begin{array}{l}\text { Tasa de mortalidad } \\
\text { neonatal }\end{array}$} & \multicolumn{2}{|c|}{$\begin{array}{l}\text { Partos atendidos } \\
\text { por personal } \\
\text { calificado }\end{array}$} \\
\hline Depto. & $\%$ & Depto. & $\%$ & Depto. & Años & Depto. & $\%$ & Depto. & $\begin{array}{l}\text { Por cada } \\
100000 \text { NV }\end{array}$ & Depto. & $\begin{array}{l}\text { Por cada } \\
1000 \text { NV }\end{array}$ & Depto. & $\begin{array}{l}\text { Por cada } \\
1000 \mathrm{NV}\end{array}$ & Depto. & $\%$ \\
\hline Paraguay & 26,6 & aazapá & 47,0 & A. Parag & 6,2 & A. Paraguay & 11,2 & A. Para & 268,6 & & 49,2 & & 45,1 & & 82,4 \\
\hline Boquerón & 48,4 & Paraaua & 46,5 & Caazapá & 6,8 & Pte. Hayes & 9,7 & & & Pte. Hayes & 34, & & 20 & San Pedro & 92,1 \\
\hline Caazapá & 51,6 & oncepción & 44,0 & Boquerón & 7,0 & Boquerón & 9,1 & Boquerón & & & 26,2 & & 15,9 & A. Paraguay & 93 \\
\hline Pte. Hayes & 63,3 & Canindeyú & 38,0 & Canindeyú & 7,4 & Ñeembucú & 6 & Amambay & 111,0 & Central & 18,6 & A. Paraná & 12,0 & Concepción & 95,9 \\
\hline
\end{tabular}

NV, nacidos vivos; A. Paraguay, Alto Paraguay; A. Paraná, Alto Paraná; Pte. Hayes, Presidente Hayes; Depto., Departamento.

Fuente: Ministerio de Salud y Encuesta Permanente de Hogares, 2017.

estratificadores de equidad e indicadores de salud. Estos corresponden al tercil 1; por lo tanto, es el grupo más desfavorecido en cada una de estas variables consideradas al estudiar las desigualdades simples. Con base en los estratificadores de equidad, los departamentos Alto Paraguay, Boquerón, Caazapá, Concepción y San Pedro son los que pueden ser considerados con mayor grado de vulnerabilidad social, ya que se encuentran en todos o casi todos los indicadores como zonas de mayor vulnerabilidad. Por otro lado, en términos de los indicadores de salud, los departamentos Alto Paraguay, Presidente Hayes y Boquerón, que pertenecen al Chaco paraguayo, son los que tienen los peores resultados en todos los indicadores de salud considerados.

\section{Desigualdades en los indicadores de la meta del ODS 3.1}

En términos de las desigualdades sanitarias en la RMM, las medidas simples tienen valores estimados de $\mathrm{BA}=72,1 \mathrm{y}$ $\mathrm{BR}=2,3$, lo cual indica que entre los terciles de departamentos con los valores de RMM más altos y más bajos existe un exceso de mortalidad equivalente a 72 muertes maternas por 100000 $\mathrm{NV}$, y que el riesgo de muerte materna en el tercil de departamentos con RMM más altas es más de dos veces el riesgo del tercil de departamentos con RMM más bajas. Por otro lado, las medidas complejas tienen valores estimados de IDPA $=86,9$ y IDRP $=3,2$, lo cual indica que, en términos absolutos, hay 87 muertes maternas más por $100000 \mathrm{NV}$ en el departamento con RMM más alta en comparación con el departamento con RMM más baja, y que en términos relativos el riesgo de muerte materna en el departamento con RMM más alta es 3,2 veces el riesgo en el departamento con RMM más baja.

Al examinar las desigualdades sociales en la RMM, se observa que las mayores desigualdades se presentan en los siguientes estratificadores de equidad: porcentaje de acceso a saneamiento mejorado (PASM) y promedio de años de estudio (PAE) (cuadro 2). Al utilizar el PASM, los valores estimados de las medidas simples son $\mathrm{BA}=30,9$ y $\mathrm{BR}=1,5$, lo cual indica que entre los terciles de departamentos con PASM más bajos y más altos existe un exceso de mortalidad equivalente a 31 muertes maternas por $100000 \mathrm{NV}$, y que el riesgo de mortalidad materna en el tercil de departamentos con PASM más bajos es 1,5 veces el riesgo en el tercil de departamentos con PASM más altos. Por
CUADRO 2. Medidas de desigualdades simples y complejas

\begin{tabular}{|c|c|c|c|c|}
\hline \multicolumn{5}{|c|}{ Indicador ODS 3.1.1: razón de mortalidad materna (RMM) } \\
\hline \multirow[t]{2}{*}{ Estratificador de equidad } & \multicolumn{2}{|c|}{ Medidas simples } & \multicolumn{2}{|c|}{ Medidas complejas } \\
\hline & $\mathrm{BA}$ & BR & IDABN & IDRBN \\
\hline $\mathrm{RMM}^{\mathrm{a}}$ & 71,3 & 2,3 & 86,9 & 3,2 \\
\hline $\begin{array}{l}\text { Porcentaje de acceso a saneamiento } \\
\text { mejorado }\end{array}$ & 30,9 & 1,5 & 45,5 & 1,8 \\
\hline Porcentaje de pobreza total & 7,7 & 1,1 & 23,8 & 1,3 \\
\hline Promedio de años de estudio & 21.0 & 1,3 & 53,1 & 2,0 \\
\hline Tasa de desempleo abierto & 10,4 & 1,2 & 39,9 & 1,6 \\
\hline \multicolumn{5}{|c|}{ Indicador ODS 3.1.2: partos atendidos por profesional calificado (PPAPC) } \\
\hline PPAPC $^{a}$ & $-6,9$ & 0,93 & $-11,1$ & 0,89 \\
\hline $\begin{array}{l}\text { Porcentaje de acceso a saneamiento } \\
\text { mejorado }\end{array}$ & $-6,3$ & 0,94 & $-9,1$ & 0,91 \\
\hline Porcentaje de pobreza total & $-4,5$ & 0,95 & $-7,9$ & 0,92 \\
\hline Promedio de años de estudio & $-4,7$ & 0,95 & -10.0 & 0,90 \\
\hline Tasa de desempleo abierto & 2,5 & 1,03 & 6,0 & 1,10 \\
\hline \multicolumn{5}{|c|}{ Indicador ODS 3.2.1: tasa de mortalidad en menores de 5 años (TMM5) } \\
\hline TMM5 & 6,9 & 1,6 & 21,1 & 3,4 \\
\hline $\begin{array}{l}\text { Porcentaje de acceso a saneamiento } \\
\text { mejorado }\end{array}$ & 5,8 & 1,4 & 9,6 & 1,8 \\
\hline Porcentaje de pobreza total & 2,5 & 1,2 & 10,7 & 1,9 \\
\hline Promedio de años de estudio & 4,8 & 1,4 & 15,5 & 2,8 \\
\hline Tasa de desempleo abierto & 4,7 & 1,3 & 9,3 & 1,7 \\
\hline \multicolumn{5}{|c|}{ Indicador ODS 3.2.2: tasa de mortalidad neonatal (TMN) } \\
\hline TMN $^{\mathrm{a}}$ & 8,6 & 2,7 & 13,7 & 3,8 \\
\hline $\begin{array}{l}\text { Porcentaje de acceso a saneamiento } \\
\text { mejorado }\end{array}$ & 3,5 & 1,4 & 6,5 & 1,9 \\
\hline Porcentaje de pobreza total & 1,1 & 1,1 & 7,3 & 2,0 \\
\hline Promedio de años de estudio & 3,5 & 1,4 & 10,5 & 3,0 \\
\hline Tasa de desempleo abierto & 3,4 & 1,4 & 7,4 & 1,9 \\
\hline
\end{tabular}

aproduce la desigualdad sanitaria.

Fuente: Ministerio de Salud y Encuesta Permanente de Hogares, 2017.

su parte, las medidas complejas estimadas son IDABN $=45,5$ y IDRBN $=1,8$, lo cual muestra que hay una diferencia de 46 muertes maternas por $100000 \mathrm{NV}$ entre los dos departamentos extremos, con PASM más bajo y más alto, y que el riesgo de muerte materna en el departamento con PASM más bajo es casi dos veces $(1,8)$ el riesgo en el departamento con PASM más alto. 
En todos los estratificadores de equidad, excepto en la tasa de desempleo abierto, las medidas absolutas simples y complejas en el PPAPC tienen valores negativos y las relativas tienen valores menores a 1 (cuadro 2). Esto es debido a que las medidas de desigualdades sanitarias y sociales comparan los valores del PPAPC en los terciles o departamentos con mayores niveles de vulnerabilidad (donde el PPAPC tiende a ser más bajo) con respecto a los de menores niveles de vulnerabilidad (donde el PPAPC tiende a ser más alto). En el caso de las desigualdades sanitarias en el PPAPC, las medidas simples obtenidas son $\mathrm{BA}=-6,9$ y $\mathrm{BR}=0,93$, lo que indica que el PPAPC es 6,9 puntos porcentuales menor en el tercil de departamentos con PPAPC más bajos en comparación con el tercil con PPAPC más altos, y que el tercil de departamentos con PPAPC más bajos presenta un $7,0 \%(100 *[0,93-1]=-7,0)$ de PPAPC menos que el quintil con PPAPC más altos. Por otra parte, las medidas complejas obtenidas son IDAL $=-11,1$ y IDRL $=0,89$, la cuales se interpretan en forma similar a las medidas simples, pero en vez de comparar el tercil de departamentos con PPAPC más bajos y el tercil con PPAPC más altos, se comparan los departamentos con el PPAPC más bajo y más alto.

En general, las desigualdades sociales en el PPAPC tienen medidas simples y complejas no elevadas, aunque los valores más grandes de estas medidas se presentan al considerar los estratificadores de equidad correspondientes al PASM y PAE (cuadro 2).

\section{Desigualdades en los indicadores de la meta del ODS 3.2}

Las desigualdades sanitarias y sociales en la TMM5 y la TMN tienen comportamientos similares en sus medidas simples y complejas. Al evaluar las desigualdades sociales en la TMM5 y la TMN, se observa que los niveles más altos de desigualdades simples se presentan en los estratificadores de equidad PASM y PAE. En términos de desigualdades complejas, las mayores desigualdades se presentan en los estratificadores de equidad correspondientes al porcentaje de pobreza total y PAE. Al considerar la desigualdad social en la TMM5 con el estratificador de equidad PAE, las medidas de desigualdades simples estimadas son $\mathrm{BA}=4,8$ y $\mathrm{BR}=1,4$, lo cual muestra que hay una diferencia de casi 5 muertes en menores de 5 años por $1000 \mathrm{NV}$ entre el tercil de departamentos con PAE más bajos y el tercil de departamentos con PAE más altos, y que hay $40 \%$ más de muertes de menores de 5 años en el tercil de departamentos con PAE más bajos (figura 2). Por su parte, las medidas complejas estimadas corresponden a IDABN $=15,5$ y IDRBN $=2,8$ (cuadro 2). Esto indica que hay un exceso de casi 16 muertes en menores de 5 años por $1000 \mathrm{NV}$ entre los departamentos extremos, con PAE más bajo y más alto, y que el riesgo de mortalidad en menores de 5 años en el departamento con PAE más bajo es casi tres veces $(2,8)$ el riesgo en el departamento con PAE más alto.

\section{DISCUSIÓN}

Congruente con otros estudios $(2,15)$, los resultados presentados aquí permiten observar que los valores nacionales en la RMM, PPAPC, TMM5 y TMN ocultan las variaciones de los valores de estos indicadores entre los departamentos de Paraguay.
FIGURA 2. Tasa de mortalidad en menores de 5 años por cada 1000 nacidos vivos por terciles de promedio de años de estudio en los departamentos de Paraguay, 2017

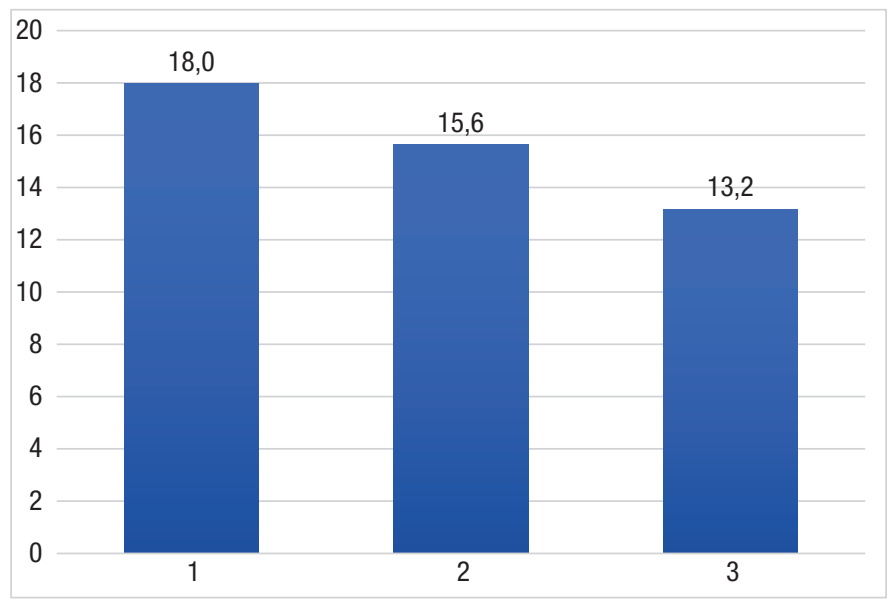

Fuente: Ministerio de Salud de Paraguay.

Este estudio evidencia la magnitud de las desigualdades sanitarias y sociales en la RMM, PAPC, TMM5 y TMN que existen entre los departamentos de Paraguay. La información producida en este trabajo permite identificar los departamentos que producen las mayores desigualdades sanitarias y sociales, lo cual debería servir de insumo a tomadores de decisiones con objeto de aplicar los mecanismos necesarios para reducir las desigualdades sanitarias y sociales entre los departamentos. Además, proporciona información sobre los estratificadores de equidad que generan estas desigualdades sociales en salud. Por otro lado, esta información puede utilizarse para desarrollar otros estudios relacionados con barreras específicas en salud que pueden afectar a departamentos con mayores niveles de vulnerabilidad social.

La RMM es un indicador que evidencia el funcionamiento del sistema de salud de un país, por lo que la muerte de una mujer por causas relacionadas con el embarazo, parto y puerperio refleja la inequidad hacia las mujeres y sus condiciones de vida. Si bien Paraguay ha disminuido la muerte materna en los últimos años, las desigualdades sociales y sanitarias al interior del país presentan diferencias significativas entre los departamentos. Esto no ocurría al analizar solo el promedio nacional; por ejemplo, la RMM en Alto Paraguay es de casi cuatro veces el promedio nacional. Existe una magnitud elevada en la desigualdad sanitaria y social en la RMM; en términos de la desigualdad sanitaria, los departamentos más afectados son Presidente Hayes, Boquerón, Canindeyú, Concepción y Amambay. Al utilizar el estratificador de equidad PASM, el cual produce la mayor desigualdad social en la RMM, los departamentos más afectados son Alto Paraguay, Boquerón, Caazapá, Concepción, San Pedro y Presidente Hayes.

La atención del parto por personal calificado es considerada una buena práctica para disminuir los riesgos durante el parto. Hay evidencia histórica y observacional de que la asistencia especializada reduce el riesgo de muerte materna. La atención de todos los partos por personal calificado otorga a todas las mujeres que padecen complicaciones potencialmente mortales una mayor posibilidad de recibir atención obstétrica de 
emergencia a tiempo (16). Según la OMS, entre 1983 y 2000, la proporción de partos atendidos por asistentes de partería cualificados se duplicó y la RMM disminuyó 50\% (17). En Paraguay, en general no se observa una gran desigualdad sanitaria ni social en este indicador.

A nivel mundial, el riesgo de morir antes de los 5 años en niños más pobres es casi dos $(1,9)$ veces el riesgo de morir en niños más ricos (18). La mortalidad neonatal en todo el mundo está descendiendo con menor rapidez que la mortalidad de niños entre 1 mes y 5 años (18); por lo tanto, la mortalidad en la niñez y más aún la mortalidad infantil, está concentrada en la etapa neonatal. Para mejorar la supervivencia infantil, se requieren intervenciones asequibles y basadas en evidencia que permitan combatir las principales enfermedades infecciosas, como por ejemplo una adecuada atención postnatal, inmunización, acceso a agua potable y adecuada nutrición (18). Las complicaciones derivadas del nacimiento prematuro (que ocasionan $35,0 \%$ de las muertes) o las complicaciones en el parto o en el nacimiento $(24,0 \%)$ precisan de intervenciones sanitarias vinculadas a la protección de la salud materna (18); por lo tanto, estos indicadores, sobre todo la mortalidad neonatal, evidencian la importancia del cuidado del binomio madre hijo. En Paraguay, las desigualdades en la TMM5 y la TMN se comportan de forma similar y dan cuenta de la necesidad de realizar esfuerzos en aquellos departamentos con mayor porcentaje de pobreza y menor promedio años de estudio. En términos de desigualdad sanitaria y social (por el estratificador PASM), los departamentos que producen las mayores desigualdades en la TMM5 y la TMN son los mismos que producen las mayores desigualdades en la RMM.

Una de las fortalezas de este artículo es el uso de medidas de desigualdades producidas al ajustar los modelos de regresión binomial negativo y logístico. Por otro lado, se han observado algunas limitaciones en este estudio, como es el caso de la falacia ecológica debido al tipo de diseño considerado; esto significa que las desigualdades entre los departamentos no pueden ser interpretadas a nivel de los individuos. Otra limitación tiene que ver con la calidad de los datos administrativos de los indicadores de salud en los departamentos. En los datos relacionados con la mortalidad, se debe tener presente que existe un porcentaje de subregistro y mala clasificación de las muertes, el cual tiende a ser diferenciado entre los departamentos; es decir, el subregistro de las muertes puede ser más elevado en los departamentos con mayor vulnerabilidad social.

Este estudio produce información basal de la magnitud de las desigualdades sanitarias y sociales en los indicadores del ODS 3.1 y ODS 3.2 entre los departamentos de Paraguay en 2017. Esto muestra que, además de medir y monitorear el valor nacional de un indicador de salud, se deben medir y monitorear las desigualdades sanitarias y sociales en salud $(1,19)$, con la finalidad de identificar las brechas y generar estrategias que permitan mejorar la salud de la mujer y el niño. Esto ayudará a tener mejores resultados en los indicadores de impacto como la mortalidad materna, neonatal y de la niñez. Por otro lado, la medición de las desigualdades sanitarias y sociales permite a tomadores de decisiones de salud utilizar información adecuada para el desarrollo de políticas de salud en los departamentos que producen las mayores desigualdades.

Si bien las desigualdades sanitarias y sociales en el PPAPC no son elevadas, aún persisten en la RMM, la TMM5 y la TMN entre los departamentos de Paraguay, las cuales deben ser reducidas. Se debe reforzar aún más el trabajo en el binomio madre-hijo en los departamentos que tienen un mayor grado de vulnerabilidad social (Alto Paraguay, Boquerón, Caazapá, Concepción y San Pedro), iniciando con un cuidado prenatal adecuado, atención del parto por personal calificado, cuidado posnatal adecuado e intervenciones de salud para el cuidado del neonato, el lactante y el niño que permitan disminuir la mortalidad materna y neonatal.

El presente estudio evidencia las grandes desigualdades en indicadores de salud que existen entre los departamentos de Paraguay. El país debería institucionalizar la medición y el monitoreo de las desigualdades. Además, se deberían establecer metas (numéricas) en los indicadores de salud del ODS 3, tanto de mejoría de los promedios nacionales, como de reducción de las desigualdades entre los departamentos. Esto permitirá rendir cuentas sobre el compromiso de "no dejar a nadie atrás" establecido en los ODS.

Los datos de los indicadores de salud y estratificadores de equidad utilizados en este estudio provienen de diferentes fuentes de información, registros administrativos y encuestas; se deben seguir fortaleciendo los sistemas de información para la salud con el objeto de proveer datos de calidad que permitan monitorear las metas establecidas en los indicadores de salud.

Agradecimientos. A la iniciativa Todas las Mujeres Todos los Niños en Latinoamérica y el Caribe (EWEC-LAC, por su sigla en inglés), al Ministerio de Salud de Paraguay y a Luis Roberto Escoto, representante de la OPS/OMS en Paraguay, por el constante apoyo a las acciones de cooperación técnica en el país.

Conflicto de intereses. Ninguno declarado por los autores.

Declaración. Las opiniones expresadas en este manuscrito son responsabilidad de los autores y no reflejan necesariamente los criterios ni la política de la RPSP/PAJPH y / o de la Organización Panamericana de la Salud (OPS).

\section{REFERENCIAS}

1. Mujica OJ, Moreno CM. De la retórica a la acción: medir desigualdades en salud para "no dejar a nadie atrás". Rev Panam Salud Publica. 2019;43:e12. Disponible en: https://doi.org/10.26633/ RPSP.2019.12.

2. Sanhueza A, Calle Roldán J, Ríos-Quituizaca P, Acuña MC, Espinosa I. Social inequalities in maternal mortality among the provinces of Ecuador. Rev Panam Salud Publica. 2017;41:e97.
3. Ministerio de la Mujer, Programa para la Cohesión Social en América latina (EUROsociAL), Entidad de la Organización de las Naciones Unidas para la Igualdad de Género y el Empoderamiento de la Mujer (ONU Mujeres). Marco para el Diseño de la Política Nacional de cuidados en el Paraguay. Disponible en: https://lac.unwomen.org/es /digiteca/publicaciones/2019/06/ documento-marco-paraguay 
4. Dirección General de Estadística de Paraguay, Encuestas y Censos. Condiciones de Vida EPHC 2009. Disponible en: https:// www.datos.gov.py / dataset/condiciones-de-vida-ephc-20092018

5. Dirección General de Estadísticas de Paraguay, Encuestas y Censos. Anuario Estadístico del Paraguay 2015. Disponible en: https:// www.dgeec.gov.py / Publicaciones / Biblioteca / anuario2015/ Anuario\%20Estadistico\%202015.pdf

6. Organización Panamericana de la Salud, Organización Mundial de la Salud. Indicadores Básicos. Situación de Salud de las Américas. 2019. Disponible en: https://www.paho.org/data/index.php/en/ indicators.html.

7. Corsi DJ, Neuman M, Finlay JE, Subramanian SV. Demographic and health surveys: a profile. Int J Epidemiol. 2012;41(6):1602-13.

8. Hancioglu A, Arnold F. Measuring coverage in $\mathrm{MNCH}$ : tracking progress in health for women and children using DHS and MICS household surveys. PLoS medicine. 2013;10(5):e1001391-e.

9. World Health Organization. Global Health Observatory's Health Equity Monitor. Geneva: WHO; 2014. Disponible en: https:/ /www. who.int/gho/health_equity/en/

10. Dirección General de Estadísticas, Encuestas y Censos de Paraguay. Encuesta Permanente de Hogares 2017. Disponible en: http:// www.dgeec.gov.py

11. Mackenbach JP, Kunst AE. Measuring the magnitude of socioeconomic inequalities in health: an overview of available measures illustrated with two examples from Europe. Soc Sci Med. 1997;44(6):757-71.

12. Schneider MC, Castillo C, Bacallao J, Loyola E, Mújica OJ, Vidaurre $\mathrm{M}$, et al. Métodos de medición de las desigualdades de salud. Rev Panam Salud Publica. 2002;12(6):398-415.

13. Harper S, King NB, Meersman SC, Reichman ME, Breen N, Lynch J. Implicit value judgments in the measurement of health inequalities. The Milbank Q. 2010;88(1):4-29.
14. Bacallao J. Indicadores basados en la noción de entropía para la medición de las desigualdades sociales en salud. Rev Cub Salud Publica. 2007;33(4)

15. Enriquez Nava MF, Esquivel Velásquez AD, Patón Sanjines M, Pooley Ayarza BC, Alarcón R, Hernández Muñoz et al. Comportamiento y desigualdades sociales en indicadores priorizados del Objetivo de Desarrollo Sostenible 3 en Bolivia. Rev Panam Salud Publica. 2020;44:e101. https://doi.org/10.26633/ RPSP.2020.101.

16. Fondo de Población de las Naciones Unidas. Mortalidad materna: el parto en buenas manos. Nueva York: FPNU; 2004. Disponible en: https://www.unfpa.org/sites/default/files/pub-pdf/mmupdate 05_spa.pdf

17. Organización Mundial de la Salud. Informe sobre la salud en el mundo 2005 - ¡Cada madre y cada niño contarán! Ginebra: OMS; 2005:65-7.

18. Fondo de Naciones Unadas para la Infancia (UNICEF). Estado Mundial de la Infancia 2016: Una Oportunidad para cada niño. Nueva York: UNICEF; 2016. Disponible en: https://www. unicef.org/spanish/publications / files/UNICEF_SOWC_2016_ Spanish.pdf

19. Organización Panamericana de la Salud. Manual para el Monitoreo de las Desigualdades en Salud, con especial énfasis en países de ingresos medianos y bajos. Washington D.C.: OPS; 2016.

Manuscrito recibido el 11 de mayo de 2020. Aceptado para su publicación, tras revisión, el 2 de julio de 2020.

\section{Health and social inequalities in maternal and child health in Paraguay}

ABSTRACT Objective. To describe health and social inequalities in maternal and child health indicators defined in Sustainable Development Goal (SDG) 3.1 and SDG 3.2 targets based on administrative data among the departments of Paraguay in 2017.

Methods. Quantitative descriptive study with ecological design. Simple gap measures and complex gradient measures based on the adjustment of negative binomial and logistic regression models were used.

Results. Fifty percent of Paraguay's departments have estimated maternal mortality ratio (MMR) values higher than the national value. The percentage of births attended by a qualified professional in the country is $98.1 \%$, with a range between $82.4 \%$ and $99.9 \%$. In 13 of 18 departments, under-five mortality rate (U5MR) is higher than the national average, ranging from 4.2 to 49.2 deaths per 1000 live births. Neonatal mortality rates (NMR) in the departments vary from 2.6 to 45.1 deaths per 1000 live births. There are major health and social inequalities in the MMR, U5MR and NMR between the departments. There are no high inequalities in the percentage of births attended by a qualified professional between the departments.

Conclusions. Paraguay needs to make significant efforts to reduce the health and social inequalities that exist in the MMR, U5MR and NMR between departments. Numerical targets must be established to improve national values and reduce inequalities in these indicators, which will allow for accountability on the commitment to "leave no one behind" established in the SDG, and will help generate strategies to improve the health of women and children in Paraguay.

Keywords Sustainable development; health status disparities; maternal mortality; infant mortality; Paraguay. 